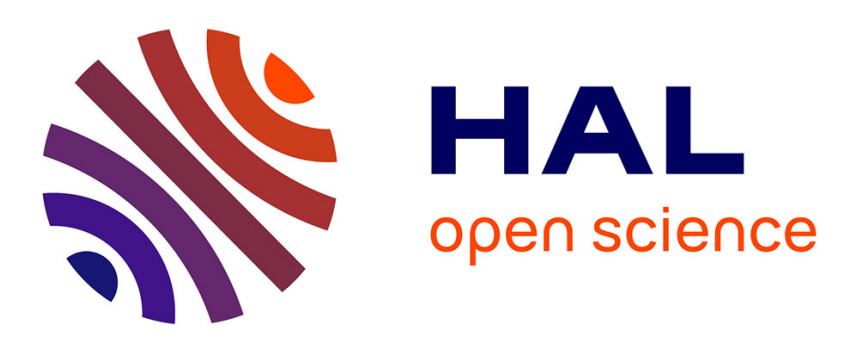

\title{
Fast quantification of avian eggshell microstructure and crystallographic-texture using two-dimensional X-ray diffraction
}

\author{
Alejandro B Rodriguez-Navarro
}

\section{- To cite this version:}

Alejandro B Rodriguez-Navarro. Fast quantification of avian eggshell microstructure and crystallographic-texture using two-dimensional X-ray diffraction. British Poultry Science, 2007, 48 (02), pp.133-144. 10.1080/00071660701302262 . hal-00545310

\author{
HAL Id: hal-00545310 \\ https://hal.science/hal-00545310
}

Submitted on 10 Dec 2010

HAL is a multi-disciplinary open access archive for the deposit and dissemination of scientific research documents, whether they are published or not. The documents may come from teaching and research institutions in France or abroad, or from public or private research centers.
L'archive ouverte pluridisciplinaire HAL, est destinée au dépôt et à la diffusion de documents scientifiques de niveau recherche, publiés ou non, émanant des établissements d'enseignement et de recherche français ou étrangers, des laboratoires publics ou privés. 


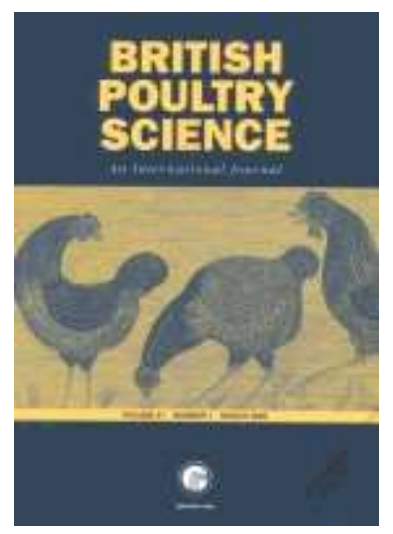

\section{Fast quantification of avian eggshell microstructure and crystallographic-texture using two-dimensional X-ray diffraction}

\begin{tabular}{|r|l|}
\hline Journal: & British Poultry Science \\
\hline Manuscript ID: & CBPS-2006-027.R2 \\
\hline Manuscript Type: & Original Manuscript \\
\hline Date Submitted by the \\
Author: & 06-Sep-2006 \\
\hline $\begin{array}{r}\text { Complete List of Authors: } \\
\text { Keywords: }\end{array}$ & $\begin{array}{l}\text { Rodriguez-Navarro, Alejandro; Universidad de Granada, } \\
\text { Departamento de Mineralogia y Petrologia }\end{array}$ \\
\hline & \\
\hline
\end{tabular}

\section{今 ScholarONE \\ Manuscript Central}


Rapid quantification of avian eggshell microstructure and crystallographic-texture using two-dimensional X-ray diffraction
A. B. Rodriguez-Navarro, Departamento de Mineralogía y Petrología, Universidad de Granada, Granada, Spain.

\author{
Short title: QUANTIFYING EGGSHELL MICROSTRUCTURE \\ Correspondence to Dr Alejandro B. Rodriguez-Navarro, Departamento de Mineralogía \\ y Petrología, Universidad de Granada, 18002 Granada, Spain. \\ Tel: +34 958240059; \\ Fax: +34 958243368; \\ E-mail: anava@ugr.es
}


Abstract. 1. It is important to quantify eggshell microstructure because it may influence eggshell mechanical properties and could be of importance for eggshell quality assessment. It also provides information about changes in eggshell associated with different hen physiological conditions.

2. This paper describes an alternative methodology which, based on 2D X-ray diffraction, allows eggshell microstructure quantification (for example, crystal size and orientation) much more efficiently than using traditional techniques (optical or scanning electron microscopy and conventional X-ray diffraction).

3. For such analyses, an X-ray diffractometer equipped with an area detector is used. Such equipment collects the diffraction pattern of the sample in one single exposure (taking a few tens of seconds), thus considerably reducing data acquisition time.

4. The two dimensional diffraction patterns obtained contain detailed information about size and crystallographic orientation of crystals forming the eggshell. This information can be automatically extracted using specially designed software.

5. Access to the software is available on a website, the address of which is given in the Materials and Methods.

6. In this paper, specific examples of how these analyses are applied are set out and recommendations for obtaining different types of microstructure information are given. In addition, the limitations of the technique are discussed.

\section{INTRODUCTION}

Eggshell is a bioceramic material consisting mainly (>95\%) of the mineral calcite $\left(\mathrm{CaCO}_{3}\right)$ and a pervading organic matrix $(1-3.5 \%)$, resulting in a composite structure which has excellent mechanical properties (Simkiss and Wilbur, 1989; Arias et al., 1993; Nys et al., 1999). The mineral part of the shell is comprised of distinct layers. The size and orientation of crystals change across these layers. Starting from the interior to the exterior are the mamillary layer (made of small spherulitic calcite crystals displaying a random orientation), the palisade layer (made of columnar calcite crystals with a preferential orientation), the vertical crystal layer and the cuticle layer (covering the outer surface). The amount of material eggshell thickness and/or density) is the main factor contributing to the mechanical properties of the eggshell. Nevertheless, there is also evidence that the structural organisation of the eggshell at different levels significantly influences its mechanical properties (Meller et al., 1973; van Toledo et al., 1982; Rodriguez-Navarro et al., 2002; Lammie et al., 2006). Ultrastructure (defined by the extend and disposition of major structural units) and microstructure (defined by the size of crystals, their shape and crystallographic orientation -or texture-) characteristics are especially important. Regarding ultrastructure, available data shows that weaker eggshells generally have a thinner palisade layer and/or a higher density of mamillary knobs than stronger eggshells (Meller et al., 1973; van Toledo et al., 1982). Regarding microstructure, weaker eggshells have, for instance, a number of defects or scratches 
that could act as a site for nucleation of cracks. Also they are formed by crystals of abnormal sizes (generally larger) and shapes which negatively affect their mechanical performance (Rodriguez-Navarro et al., 2002; Ahmed et al., 2005). Additionally, the preferential orientation of crystals has a strong influence on eggshell mechanical properties (Rodriguez-Navarro et al., 2002). Eggshells having a weak orientation are stronger than those highly oriented. The dependence on crystallographic orientation is probably due to the fact that calcite is easily cleaved along specific crystallographic directions.

Most of these data have been collected using optical or scanning electron microscopy (OM, SEM) or conventional X-ray diffraction for crystal orientation characterization (Meller et al., 1973; van Toledo et al., 1982; Sharp and Silyn-Robert, 1984; Rodriguez-Navarro et al., 2002). While these techniques provide detailed information of the structural organisation, they are time-consuming and do not always provide quantitative information to characterise the microstructure (for example,. average crystal size, shape and orientation). Furthermore, microstructural changes can be very subtle and in most cases can only be detected after statistical analyses of a sufficiently large number of samples. More efficient methodologies are needed (Ahmed et al., 2005). This paper describes an alternative methodology which, based on 2D Xray diffraction, allows eggshell microstructure quantification much more efficiently than using traditional techniques. For such analyses, a X-ray diffractometer equipped with an area detector instead of a point detector is used. Such equipment collects the diffraction pattern of the sample in one single exposure (taking a few tens of seconds), reducing considerably the time for data acquisition. In addition, 2D diffraction patterns contain detailed information about size and crystallographic orientation of crystals forming the eggshell (Cain and Heyn, 1964; Ahmed et al., 2005). This information can be extracted using appropriate analytical software.

In this paper, specific examples of how these analyses are applied are set out and ways of obtaining different types of microstructure information are described, together with specific recommendations for experiments and some limitations of the technique. In addition, software specially designed to automate data analysis and which allows the rapid extraction of information from $2 \mathrm{D}$ diffraction patterns is described. The primary aim of the paper is to make known the availability and applicability of this methodology to poultry scientists needing to characterise a large number of samples for eggshell quality studies. 


\section{MATERIALS AND METHODS}

\section{Samples}

Eggshell samples from different bird species including laying hen, ostrich, Egyptian vulture and partridge, representing a large range of microstructure characteristics (different crystal sizes and orientation) and thicknesses were selected.

\section{Optical microscopy}

Radial thin-sections $(<30 \mu \mathrm{m})$ of eggshells were prepared for microstructureal analysis by optical microscopy (OM). A small piece of shell cut from the equatorial region of each egg was embedded in resin and double polished following methodology described elsewhere (Rodriguez-Navarro et al., 2002). Eggshell microstructure in thin-sections was analysed by OM with cross-polarised light (Olympus SZ, Japan).

\section{X-ray diffraction}

\section{Sample setting}

Pieces (about 1x1 cm) were cut from each eggshell sample, mounted using clay-dough and set on a sample holder of a single crystal diffractometer equipped with an area detector (2D-XRD; D8 SMART APEX, Bruker, Germany). Two types of settings were used: 1) transmission and 2) reflection. 1) For transmission experiments, samples were mounted so that their outer shell surface faced the area detector and the inner surface faced the incident X-ray beam (Figure 1a). 2) For reflection experiments, samples were mounted so that the X-ray beam was incident at 10 degrees (omega angle $=10$ ) on the shell outer surface and the area detector was set at a receiving 2 theta angle equal to 20 degrees (Figure 1b). Table 1 summarises the experimental conditions used for diffraction analyses.

\section{Two-dimensional diffraction patterns}

In a diffraction experiment, crystals will reflect the X-rays if they have a set of (hkl) crystallographic planes oriented so that they fulfil the Bragg condition (Cullity, 1977), producing a reflection spot denoted as hkl. The (hkl) are the Miller indices defining the crystallographic plane. These planes have a constant orientation and interplanar spacing $\left(\mathrm{d}_{\mathrm{hkl}}\right)$ across a crystal. Typically, a sample constituted by randomly-oriented crystals (such as a powder) will produce a diffraction pattern consisting of concentric rings (known as Debye-Scherrer rings) each one associated with a different set of (hkl) crystallographic planes with different $d_{h k l}$ interplanar spacing. Specific sample 
characteristics (crystal size and orientation) will modify the appearance of this diffraction pattern. For instance, crystal size affects the pattern because diffraction rings are formed by the superposition of reflection spots produced from individual crystals. For polycrystalline materials constituted by very small crystals (compared to the X-ray beam cross-section), the beam illuminates a large number of crystals and a pattern forms consisting of continuous rings. As the size of crystals increases, the number of crystals illuminated become insufficient for the entire ring to be reflected and rings become spotty (Rodriguez-Navarro et al., 2006). On the other hand, as crystal sizes increase, the intensity of reflection spots increases accordingly. Thus, from the intensity of the reflection spots of a transmission pattern, crystal size can be determined using methodology described in detail elsewhere (Rodriguez-Navarro et al., 2006; RodriguezNavarro et al., in press). Figure 3 illustrates the effect of crystal size and orientation on the diffraction patterns of eggshells having different microstructural characteristics.

Preferred crystal orientation and Psi scans

Preferential orientation of crystals also modifies the 2D diffraction pattern. For instance, depending on the orientation of crystals, some of the rings will disappear or only a small fraction of them will be displayed. In those cases, the preferential orientation of crystal prevents some specific planes from diffracting because they are oriented in such a way that they can not fulfil the Bragg condition. Generally, calcite crystals forming the eggshell are preferentially oriented with their $\boldsymbol{c}$-axis perpendicular to the shell surface and rotated around it. Thus, (hkl) planes other than (001), which is perpendicular to the $\boldsymbol{c}$-axis, will be distributed describing a cone with their $\boldsymbol{c}$-axis as their cone axis. In transmission experiments, as shown in Figure 1a, this disposition of planes results in the formation of homogeneously distributed spotty rings (one hkl ring is shown; Figure 1A). Also, as the $c$-axis is parallel to the incoming X-ray beam, only (hkl) planes sustaining a relatively small angle with the $c$-axis will be reflected around a hkl Debye ring such that depicted in the figure 1a.

In a reflection setting, as shown in Figure $1 b$, the (001) plane is almost parallel to the incident X-ray beam while the $c$-axis is oriented perpendicular to it. In this case, the (001) will be reflecting and will produce a reflection spot (i.e., 006) at 90 degrees from it and from the eggshell surface (which is nearly parallel to (001) planes). Other (hkl) planes ((104) plane) will be distributed describing a cone with their $\boldsymbol{c}$-axis as their cone axis. Because of their disposition (104) planes will produce two 104 reflection spots, one at either side of a 006 reflection, forming an angle equal to interplanar angle 
between the (001) and (104) planes as shown in Figure 1b, where the spots are concentrated in small sections of the ring at either side where the 006 reflection is displayed (Figure 1b and Figure 3a3). It is also shown in this figure that when registering a theta-2theta scan using a point detector and a conventional diffractometer, only the 006 reflection would be detected. In general, due to misalignment of the $\boldsymbol{c}$-axes of crystals, instead of a single spot, several spots falling into a fraction of the ring or arc, around this position, will be displayed. The spots will became concentrated into smaller section of the ring as the preferential orientation of crystals increases. Thus, detailed information about crystal orientation can be deduced by analysing the intensity profile along a Debye ring as a function of Psi angle (in the corresponding Psi scan). For instance, from the angular spread of this arc, or the breadth of the band in a Psi scan, the scattering in the orientation of crystal $\boldsymbol{c}$-axes can be determined and thus the degree of crystal alignment along this crystallographic direction. In the case of a spotty Debye ring, the Psi scan would display a discontinuous band consisting in an array of peaks like the one displayed in Figure 2, instead of a continuous one like that shown in Figure 4b. In this case, the breadth of the band is calculated as that of a Gaussian envelope.

Theta-2theta linear scan

A theta-2theta linear scan, equivalent to that collected with a conventional diffractometer, can be calculated from the 2-D diffraction pattern by integrating the intensity of pixels equidistant to the pattern centre (direct X-ray beam) and plotting the resulting intensity versus the distance to the centre (previously converted to 2-theta values). Information regarding mineral composition and preferential orientation can be inferred from the calculated linear scans. The intensity of each hkl peak is proportional, among other factors, to the number of irradiated crystals having $\{\mathrm{hkl}\}$ crystal planes in Bragg orientation (Cullity, 1977). A quantitative estimation of the degree of crystal orientation can be obtained from the 2 Theta scans by calculating the ratio between the integrated intensities of the 006 and 104 reflections (in case the pattern was measured using a reflection setup), or that between 110 and 104 (in a transmission setup). Both ratios increase as the preferential orientation of crystals increases and can be used as an orientation index. Other indexes can be defined to measure the preferential degree of orientation from these scans (Sharp and Silyn-Robert, 1984; Rodriguez-Navarro et al., 2002). For instance, the ratio between the integrated peak areas of hkl peaks from the intact and ground shell (in which crystals have a random orientation) provides also a measure of the relative orientation of crystals in the intact shell. 


\section{Software description and use}

We have developed specially-designed Windows software (XRD2DScan; available at www.ugr.es/ anava/xrd2dscan.htm) using Borland Delphi ${ }^{\circledR}$ that automatically processes the information contained on $2 \mathrm{D}$ diffraction patterns. At this website there is available a complete description of features and use of the software. The typical interface of the program is shown in Figure 2. First, the user has to load the output data files, generated by the diffractometer, containing the pixel intensities measured by the area detector. The $2 \mathrm{D}$ diffraction pattern is displayed and the theta-2theta scan is calculated. Then, the user can select the Debye ring to be analysed (for instance, by clicking at either side on a reflection peak displayed in the theta-2theta scan). Then, the software finds pixels in the $2 \mathrm{D}$ pattern belonging to the selected ring, calculates and plots the intensity of pixels as a function of Psi angle (Psi scan), searches for peaks along the intensity profile of the calculated Psi scan (with intensities above a certain intensity threshold defined by the user) and calculates their integrated intensities. In addition, the program reports the following information in a logbook: a) sample name, b) 2theta angular range of the Debye ring selected, c) psi angle and intensity of each peak found, d) total number of peaks in the ring, d) average intensity of peaks. This information can also be automatically saved to an ASCII file. Later, crystal sizes can be determined from these values (especially from peak intensities) using a previously determined calibration curve, relating for instance average peak intensities and crystal sizes such those determined by Rodriguez-Navarro et al. (2006; in review). In this study, average peak intensities were determined for the Debye ring associated with the 104 calcite reflection using a 2D diffraction pattern collected with a transmission setup. Prior to that, the user needs to set the value of several parameters relevant to this analysis: these are listed in Table 2. In addition, for crystal orientation quantification, the breadth of the band in Psi scan was measured by selecting interactively a Psi angular range covering the band region (by clicking with the mouse at either side of the band while holding A key pressed). Then, the software calculates and reports in the logbook the angular breadth at half maximum (FWHM) of the band and its integrated intensity.

\section{RESULTS}

Figure 3 illustrates the effect of eggshell microstructure characteristics (crystal size and degree of orientation) in the 2-D diffraction patterns. Figures 3a1, b1, c1 and d1 are microphotographic views of eggshell thin-sections from different avian species under 
cross-polarised light: a1) ostrich, b1) Egyptian vulture (Neophron percnopterus), c1) hen, and d1) partridge. They are all composed of columnar calcite crystal units (palisades), arising from spherulitic aggregates of smaller calcite crystals (mamillary cores) anchored on to the eggshell membranes. The columnar calcite crystal units (palisades) show varying degrees of light extinction due to differences in their orientation. It can be seen in these microphotographs that average crystal sizes and the degree of crystal orientation vary from one eggshell to another. Note that columnar unit sizes in these eggshells increase from partridge to vulture eggshell. On the other hand, crystal orientation increases according to the following sequence: partridge, hen, vulture and ostrich eggshells. Figure $3 \mathrm{a} 2, \mathrm{~b} 2, \mathrm{c} 2, \mathrm{~d} 2$ are transmission diffraction patterns of these eggshells and Figure 3a3, b3, c3, d3 are reflection diffraction patterns of the same eggshells. In both cases, the diffraction patterns are constituted by concentric spotty rings (Debye ring), each one associated with a different set of (hkl) crystal planes. As the average crystal size increases, the number of crystals illuminated by the beam decreases as well as the number of reflection spots shown in the pattern. For instance, partridge eggshell is constituted of smaller crystals than those of hen eggshell, and therefore the diffraction pattern of the former displays a greater number of reflection spots. Also, as crystal sizes increase, peak intensities increase.

Regarding crystal orientation, patterns shown in Figure 3d2 and $\mathrm{d} 3$ (partridge eggshell) are characteristic of a calcite sample with random orientation in which all possible rings are displayed. An increased crystal orientation (from partridge to ostrich eggshells) shows different effects in the transmission and reflection patterns. In the reflection patterns, as crystals became more preferentially oriented (with their $c$-axis aligned and oriented perpendicular to the shell), the diffraction pattern changes accordingly; first some of the rings disappear (hen eggshell; Figure 3c3) and later the remaining rings became fragmented (vulture eggshell; Figure 3b3) and finally reflecting spots group until forming continuous arcs (ostrich eggshell; Figures $3 \mathrm{~b} 3$ and 3a3). As shown in Figure 1b, the $c$-axis is oriented perpendicular to the incident X-ray beam and the (001) planes are almost parallel to the incident X-ray beam and will produce reflection spots (006) at 90 degrees from it (Figure 3a3). Any other (hkl) plane ((104) plane) will be distributed describing a cone with their $\boldsymbol{c}$-axis as their cone axis and will produce 104 reflection spots falling in a small fraction of the 104 ring, located at either side of a 006 reflections (Figure 3b3). As crystal orientation increases further, individual spots merge and form continuous arcs (Figure 3a3). In a transmission pattern, 
these changes are not as evident until crystals became highly aligned (ostrich eggshell; Figure 3a2). Then, some of the rings disappear and only those associated with planes parallel (110), or sustaining a small angle $(113,202)$, with the $c$-axes remain. As explained earlier and illustrated in Figure 1a, calcite crystals forming the eggshell became preferentially oriented with their $\boldsymbol{c}$-axis perpendicular to the shell surface and rotated around it. Thus, in a transmission experiment, as shown in Figure 1a, the $c$-axis is parallel to the incoming X-ray beam and only (hkl) planes sustaining a small angle with the $c$-axis will be reflecting around a hkl Debye ring.

The trends in the evolution of crystal size and orientation observed in eggshells by $\mathrm{OM}$ can be better appreciated in Table 3. In this Table values for the microstructure parameters for the different eggshells calculated using OM and 2D-XRD are reported. In particular, the average integrated intensity of peaks in the Debye ring associated with 104 calcite reflections for different eggshell types are given. Peak intensities increase from partridge, hen to vulture, following the same sequence in which crystal unit sizes (measured using OM) increase. Peak intensities could not be determined for ostrich eggshell because individual peaks were not resolvable due to the marked orientation of crystals in this eggshell. Table 3 also reports quantitative data of crystal orientation in the different eggshells, determined by 2D-XRD. The different parameters quantifying the crystal orientation (angular breadth; $\mathrm{I}_{110} / \mathrm{I}_{104} ; \mathrm{I}_{006} / \mathrm{I}_{104}$ ) were calculated for the different eggshell types and all follow the same trend. The angular breadth (the most sensitive parameter) decreases in the sequence partridge, hen, vulture and ostrich eggshell. The low value in the case of ostrich eggshell $\left(13.5^{\circ}\right.$ degrees $)$ is indicative of the narrow spread in the orientation of crystals. Conversely, large values of angular breadth as found in partridge and hen eggshells are indicative of an almost random orientation of crystals in these materials.

Plots displayed in Figure $4 \mathrm{a}$ and $\mathrm{b}$ are Psi scans showing the variation of the reflected intensity along the 104 Debye ring in 2D diffraction patterns as a function of Psi angle. The Psi scan shown in Figure 4a is from a partridge eggshell (constituted of crystals displaying a minor degree of preferential orientation) and consisting of a series of peaks of similar intensity coming from individual crystals. A calibration curve for crystal sizes from the intensities of peaks displayed in Debye rings (Figure 4a) has been previously calculated using eggshells with known crystals sizes (Figure 4c; RodriguezNavarro et al., in review). The relationship between crystal sizes and peak intensities was found to be linear $\left(\mathrm{CUW}=29.150+610^{-5} \mathrm{TA} ; R=0.96 ; \mathrm{N}=11\right)$, where CUW is the 
width of columnar calcite crystals and TA is the total integrated peak intensities in the main Debye rings being equal to A104 + A110 + A113 + A202 + A 108, where Ahkl is the average integrated intensity of peaks in different hkl Debye rings associated with the strongest $5 \mathrm{hkl}$ reflections of calcite. Error in the estimation of crystal sizes using this methodology and calibration curve was estimated to be about $5 \%$ (Rodriguez-Navarro et al., in review). Similar calibration curves have been calculated for other materials (abrasive powders of graded crystal sizes; Rodriguez-Navarro et al., 2006). Where this calibration step is not done only qualitative information about crystal size is obtained.

Figure $4 \mathrm{~b}$ is the intensity profile of an ostrich eggshell (constituted of crystals displaying a high degree of preferential orientation) for the 104 Debye ring showing two continuous bands or broad peaks. In the latter case, because all crystals share the same orientation, they reflect in the same portion of the Debye ring and peaks from individual crystals are not resolved but instead merge in a broad band. The small angular breadth of the band in the Psi scan of ostrich eggshell (about $19^{\circ}$ ) is indicative of the narrow spread in the crystal orientation.

Similarly, the effect of crystal orientation is also manifested in the calculated theta-2theta scans. Figure 5 illustrates the effect of an increasing preferential orientation of crystals forming the eggshells on this type of linear scans. As a preferential crystal orientation develops, some of the hkl peaks lose intensity and finally disappear (Figure $5 \mathrm{~A} 2$ and 5B2). Changes were more evident and different in the linear scans measured using a reflection setting than in those collected using a transmission setting. In the reflection pattern of highly-oriented ostrich egghell (Figure 5 A2), 006 and 104 reflections are strongly reinforced. In the transmission pattern of ostrich eggshell (Figure $5 \mathrm{~A} 1$ ), the strongest calcite 104 reflection is almost absent, while the lower intensity 110 reflection is strongly reinforced. In the case of measurements using a reflection setting (Figure 5A2), the hkl peaks disappearing are those associated with (hkl) planes oriented parallel to, or forming a small angle with the $c$-axis, (110, 113, 202 reflections), which were wrongly oriented to diffract. On the contrary, peak reflections associated with planes oriented parallel to the beam and perpendicular to the $c$-axis are reinforced $(006,108)$. Conversely, in the transmission setting (Figure 5A1) those which are lost are associated with planes forming a high angle with $c$-axis and with the incident X-ray beam $(104,108,116)$. A quantitative estimation of the degree of crystal orientation can be obtained from the 2 Theta scans by calculating the ratio between the integrated intensities of the 006 and 104 reflections (in case the pattern was measured 
using a reflection setup), or that between 110 and 104 (in a transmission setup). Both indexes were calculated for the different eggshell types analysxed and their values are reported in Table 3. They follow the same sequence of the angular breadth though the latter parameter represents a better estimation of the degree of orientation.

\section{DISCUSSION}

The avian eggshell is a highly complex material, composed of calcite crystals that display an anisotropic shape and varying sizes and degrees of preferential orientation (Nys et al., 1999; Rodriguez-Navarro et al., 2002). Microstructure characteristics (crystal size and orientation) can vary notably from one eggshell to another even within the same species. Quantification of microstructural parameters is important to understand changes in the eggshell due to multiple processes (hen ageing, moulting, diet, pollution) and to determine which factors caused those changes (Silyn-Robert and Sharp, 1986; Rodriguez-Navarro et al., 2002; Ahmed et al., 2005). Furthermore, studying microstructural organization is important to understand eggshell mineralisation itself.

The microstructural information about eggshells obtained by $\mathrm{OM}$ is highly detailed regarding size, shape and orientation of crystals. However, this data is limited to a 2D section of a very small area (at x10 field of view approximately $400 \times 400 \mu \mathrm{m}$, covering only about $20-30$ crystals). It is very difficult to automate measurements by means of image analysis. The methodology is further complicated by the fact that sample preparation for OM takes several hours and is not practical for more than a few samples. SEM observation is faster but mostly gives information about the eggshell ultrastructure (organisation and extent of different eggshell layers). It gives limited crystallographic information because crystal boundaries are not always visible (then crystal size can not be determined) and does not provide unambiguous information about crystallographic orientation. 2D X-ray diffraction technique provides more accurate quantitative information of eggshell microstructure (see Table 3). In particular, from the $2 \mathrm{D}$ diffraction pattern of a sample, a wealth of information regarding both crystal sizes and orientation can be automatically extracted using our software which allows the determination of different parameters (average peak intensities and breadth of bands in Debye rings, crystal orientation) able to quantify eggshell microstructure as demonstrated through this study. The methodology described here has been validated in previous studies in which different types of materials were analysed (eggshells, abrasive 
powders) (Ahmed et al., 2005; Rodriguez-Navarro et al., 2006). In a previous study this technique allowed the detection of subtle but significant differences in hen eggshell microstructure associated with moulting, which were apparently responsible for changes in mechanical properties (after moulting, hen eggshell mechanical properties improved without any significant variation of their thicknesses; Ahmed et al., 2005). Thus, the technique is very sensitive allowing the quantification of small changes in eggshell microstructure even between samples belonging to the same species. Furthermore, because data collection is fast, it is possible to analyzs a large number of samples for studies requiring statistical analyses and to relate other variables such as age, or diet, etc to eggshell microstructure or mechanical characteristics.

The following subsections discuss in detail the influence of various experimental variables when determining different types of microstructural information (crystal sizes and preferential orientation) as well as specific recommendations for analyses using this methodology.

\section{Determination of crystal sizes}

\section{Collimator diameter}

There is a valid range in which the size of crystals can be determined accurately using this methodology (5\% error; Rodriguez-Navarro et al., 2006; 2007 in review). This range depends on both the size of the X-ray beam used and the size of crystals to be measured. Typically, crystals need to be a few times smaller than the beam diameter, in order to illuminate a sufficient number of crystals, but not smaller than 1/100 times, otherwise there will be too many crystals diffracting and their associated reflections would merge and would not be resolvable. The valid range for the size of the collimator used $(0.5 \mathrm{~mm}$ in diameter) is from about 5 to $100 \mu \mathrm{m}$ (Rodriguez-Navarro et al., 2006), which is well suited for the typical crystal sizes in avian eggshells (about $50 \mu \mathrm{m}$ ). Nevertheless, some eggshells have smaller crystals (outer layer of emu and guinea fowl eggshells). On the other hand, eggshells such that of the ostrich, which consists of highly oriented crystals, cannot be measured either, because crystals of similar crystallographic orientation reflect in the same section of the Debye ring and their reflections are not resolvable. Thus, some information regarding the type of sample microstructure must be known if crystal sizes are to be correctly determined by $2 \mathrm{D} X$ ray diffraction. In the case of eggshells, an appropriate beam diameter would be at least $0.5 \mathrm{~mm}$ or better 0.8 to $1.0 \mathrm{~mm}$. 


\section{$X$-ray wavelength}

Depending on their wavelength, X-rays will be more or less strongly absorbed. Most commonly used X-ray sources are $\mathrm{Cu}$ and Mo X-ray tubes, which produce $\mathrm{Cu} \mathrm{K} \alpha \underline{\text { and }}$ Mo K $\alpha$ radiation with a wavelength of 1.54 and 0.71 Á, respectively. X-rays produced by $\mathrm{Cu}$ tubes are not appropriate for transmission experiments because almost all its intensity $(99 \%)$ will be absorbed in a short distance $(100 \mu \mathrm{m})$. X-rays produced by Mo tubes are much more penetrating and suitable for transmission experiments. However, eggshell thickness should not greatly exceed about $450 \mu \mathrm{m}$ otherwise the $\mathrm{X}$-rays will be strongly absorbed and diffracted intensities would decrease greatly. Thus, ostrich eggshells are too thick ( $>1 \mathrm{~mm}$ ) to be measured. In addition, it is necessary to correct the effect of absorption, especially if there is large variation in eggshell thicknesses among samples (Rodriguez-Navarro et al., 2007). The corrected intensities are calculated as $\mathrm{I}_{\text {corrected }}=\mathrm{I}_{\text {measured }} \exp (\mu t)$, where $\mu$ is the linear absorption coefficient of calcite $\left(1 / 450 \mu \mathrm{m}^{-1}\right)$ and $t$ is the eggshell thickness in microns when using Mo radiation. In reflection measurements, both $\mathrm{Mo} \mathrm{K} \alpha$ and $\mathrm{Cu} \mathrm{K} \alpha$ radiation can both be used. Nevertheless, absorption effects should be considered because only the crystals in the outer layer will contribute to the diffraction pattern. The thickness of the contributing layer will become smaller as the X-ray radiation used is less penetrating.

\section{Sample setting transmission or reflection}

Regarding the type of setting, transmission experiments are recommended for crystal size determination because intensities can be more precisely determined. When very thick eggshells (>1 mm) are measured and it is not possible to use a shorter wavelength than Mo, a reflection setting is recommended.

\section{Reflections to measure}

Debye rings associate with the strongest reflections should be measured. In the case of eggshells, the strongest calcite reflections are 104, 110, 113, 202, 108, and 116. From them, the number of peaks and reflections intensities can be measured. These data can be analysed individually or combined to improve representation and reduce data variability. The use of more than one ring is recommended to minimise the influence of any preferential orientation of crystals.

\section{Degree of orientation of crystals}

Collimator diameter 
The same factors apply as above - for eggshells, an appropriate beam diameter would be 0.8 to $1.0 \mathrm{~mm}$.

\section{$X$-ray wavelength}

In order to determine the orientation of crystals both $\mathrm{Mo} \mathrm{K} \alpha$ and $\mathrm{Cu} \mathrm{K} \alpha$ radiation can be used. Absorption effects should be considered because only the crystals of the outer layer contribute to the diffraction pattern. The thickness of the contributing layer will decrease as the X-ray radiation used is less penetrating $(\mathrm{Cu} \mathrm{K \alpha})$. The latter type of radiation is useful for studying just the orientation of crystals at the surface.

Sample setting transmission or reflection

Because the orientation distribution has a cylindrical symmetry around the shell, normal reflection experiments are more informative than transmission experiments when determining the preferential orientation of crystals making the eggshell (see Figure 1 and accompanying text).

\section{Reflections to measure}

The disposition and width of maxima along a Psi scan of a specific hkl Debye ring provides information about the tilting of the associated [hkl] crystallographic directions and the extent of preferential orientation of crystals along this direction. Typically, in eggshells, calcite crystals are preferentially oriented with their $\boldsymbol{c}$-axes aligned and oriented perpendicular to the outer surface, though preferential orientations along other directions ([10.4]) are possible. Thus, for shells, the most informative reflections are 006, 104, 108 and 110. For instance, where crystals are preferentially oriented with their $\boldsymbol{c}$-axes aligned, the Debye ring associated with the 006 reflection will display a broad maximum at $90^{\circ}$ relative to the shell surface (Figure $1 \mathrm{~b}$ and accompanying text). In the case of the 104 reflection, two bands will be displayed. Quantitative information can be obtained by measuring the angular spread (FWHM $\chi$ ) of a band in a Psi scan for a given hkl reflection or Debye ring. This parameter will be a measure of the scattering in the orientation of associated [hkl] crystallographic directions.

In conclusion, the use of 2D X-ray diffraction technique displays several advantages in the quantification of eggshell microstructure over other techniques such as OM, SEM, and conventional XRD. No sample preparation is required and all the information regarding crystals illuminated by the beam is integrated into one single pattern. The number of crystals contributing to the diffraction pattern is large allowing for more accurate statistics. Most important is that it is fast (data collection for 2D 
diffraction patterns takes only 20 s per sample) and our software fully automates data analyses. Depending on the type of microstructural information needed, a particular experimental setting and/or radiation is more appropriate than others. For instance, to determine crystal sizes it is better to use the transmission setting using Mo Ka radiation, whereas for determining crystal orientation the reflection setting is better. Using specific examples, the calculation of several parameters to quantify eggshell microstructure characteristics is described. Finally, this type of analysIs requires diffractometers equipped with area detectors, not a serious limitation because nowadays they are available in many laboratories.

\section{Acknowledgements}

This study was funded by grant REN2003-07375 (Spanish Government) and the Programa Ramon y Cajal (Spanish Government). The author is grateful to Agustin Rueda Montes (U. Granada) for the preparation of petrographic thin-sections of eggshell samples, to Yves Nys and Joel Gautron (INRA, France) for providing eggshell samples, and to Dr. Jose Romero Garzon (U. Granada) for his technical assistance during XRD analyses. The author is grateful for the useful comments and corrections of an anonymous referee.

\section{References}

AHMED, A.M.H., RODRÍGUEZ-NAVARRO, A.B., VIDAL, M.L., GAUTRON, J., GARCÍA-RUIZ, J.M. \& NYS, Y. (2005) Changes in eggshell mechanical properties, crystallographic texture and in matrix proteins induced by moult in hens. British Poultry Science, 46: 268-279.

ARIAS, J. L., FINK, D. J., XIAO, S. Q., HEUER, A. H. \& CAPLAN, A. I. (1993) Biomineralization and eggshells: cell-mediated acellular compartments of mineralized extracellular matrix. International Review of Cytology, 145: 217-250.

CAIN, C.J. \& HEYN, A.N.J. (1964) X-ray diffraction studies of the crystalline structure of the avian eggshell. Biophysical Journal, 4: 23-39.

CULLITY, B.D. (1977) Elements of X-ray Diffraction (New York, Addison-Wesley). LAMMIE, D., BAIN, M.M., SOLOMON, S.E., \& WESS T.J. (2006) Scanning microfocus small angle X-ray scattering study of the avian eggshell. Journal of Bionic Engineering, 3: 11-18. 
MELLER, R., BAKER, R.C. \& SCOTT, M.L. (1973) Effect of hen egg-shell and other calcium sources upon egg-shell strength and ultrastructure. Poultry Science, 52: 141147.

NYS, Y., HINCKE, M.T., ARIAS, J.L., GARCIA-RUIZ, J.M., \& SOLOMON, S.E. (1999) Avian eggshell mineralization. Poultry and Avian Biology Reviews, 10: 143-166. RODRIGUEZ-NAVARRO, A., KALIN, O., NYS, Y., \& GARCIA-RUIZ, J.M. (2002) Influence of microstructure on the shell strength of eggs laid by hens of different ages. British Poultry Science, 43: 395-403.

RODRIGUEZ-NAVARRO, A.B., ALVAREZ-LLORET, P., ORTEGA-HUERTAS, M., RODRIGUEZ-GALLEGO, M. (2006) Automatic crystal size determination in the micrometer range from spotty $\mathrm{X}$-ray diffraction rings of powder samples. Journal of the American Ceramic Society, 89: 2232-2238.

RODRIGUEZ-NAVARRO, A.B., YEBRA, A., NYS, Y., \& GARCIA-RUIZ, J.M. Analysis of avian eggshell microstructure using X-ray area detectors. European Journal of Mineralogy (in press).

SHARP, R.M.\& SILYN-ROBERT, H. (1984) Development of preferential orientation in the eggshell of the domestic fowl. Biophysical Journal, 46: 175:180.

SILYN-ROBERT, H. \& SHARP, R.M. (1986) Crystal growth and the role of the organic network in eggshell biomineralization. Proceedings of the Royal Society of London, B227: 303-324.

SIMKISS, K, \& WILBUR, K.M. (1989) Biomineralization: Cell Biology and Mineral Deposition. (San Diego, Academic Press).

VAN TOLEDO, B., PARSONS, A.H., \& COMBS, G.F. (1982) Role of ultrastructure in determining eggshell strength. Poultry Science, 61: 569-572. 


\section{TABLES}

TABLE 1. Experimental conditions for diffraction experiments.

\begin{tabular}{|l|l|}
\hline Diffractometer & D8 Bruker, Germany \\
Detector & SMART APEX CCD \\
Radiation & Mo Ka \\
Acceleration voltage & $50 \mathrm{kV}$ \\
Filament current & $30 \mathrm{~mA}$ \\
Collimator diameter & $0.5 \mathrm{~mm}$ \\
Collimator length & $170 \mathrm{~mm}$ \\
Exposure time & $20 \mathrm{sec}$ \\
Distance to detector & $60 \mathrm{~mm}$ \\
\hline
\end{tabular}

TABLE 2. Values of parameters set to calculate peak intensities in the 104 Debye ring using XRD2DScan software.

\begin{tabular}{lc}
\hline Parameters & Value \\
\hline 2Theta integration range (deg.) & $12.890-14.280$ \\
Background substraction & True \\
2Theta step (deg.) & 0.1 \\
Psi step (deg.) & 0.5 \\
Roll width (deg.) & 2.0 \\
Peak width (deg.) & 2.0 \\
Minimum peak intensity (counts) & 2000 \\
\hline
\end{tabular}

TABLE 3. Egshell microstructure characteristics determined by OM (thickness, columnar unit width [CUW]) and by $2 D-X R D$ (A104 [T]; band breadth [R]; $I_{110} / I_{104}$ [T]; $\left.I_{006} / I_{104}[R]\right) . R$ and $T$ within brackets indicate whether a transmission or reflection pattern was used to calculate these parameters.

\begin{tabular}{lllcccc}
\hline Eggshell & Thickness $(\mu \mathrm{m})$ & CUW $(\mu \mathrm{m})$ & $\mathrm{A}_{104}($ counts) & Band breadth (deg.) & $\mathrm{I}_{110} / \mathrm{I}_{104}$ & $\mathrm{I}_{006} / \mathrm{I}_{104}$ \\
\hline partridge & 250 & $35 \pm 14$ & $57571 \pm 12078$ & 108.9 & 0.28 & 0.00 \\
hen & 350 & $74 \pm 33$ & $127340 \pm 104706$ & 57.3 & 0.54 & 0.00 \\
vulture & 450 & $90 \pm 40$ & $135098 \pm 147792$ & 30.5 & 1.66 & 0.07 \\
ostrich & 1900 & $55 \pm 25$ & $\mathrm{NA}$ & 13.5 & 20.60 & 0.52 \\
\hline
\end{tabular}




\section{Figure Captions}

FIGURE 1. Experimental set-up for diffraction analysis of eggshell samples. A) Transmission setting. B) Reflection setting. In the reflection setting there is a shadow area in the detector as the sample partially absorbs the X-rays. For comparison, in the area detector is also outlined the scanning direction for registering a theta-2theta scan using a point detector and a conventional diffractometer, which would only detect the 006 reflection and not the 104 one. In the insets there are shown the relative orientation of planes with respect to the incident X-ray beam and calcite crystals with typical orientation in both types of settings.

FIGURE 2. Interface of data analysis software xrd2dscan. The main window displays on the left side a $2 d$ diffraction pattern and at the bottom the calculated theta-2theta linear scan. The selected Debye ring is delineated by blue lines in both the $2 d$ pattern (as section of ellipses) and in the theta-2theta scan (as vertical lines). There is another superimposed window displaying a Psi scan or the intensity profile along the selected Debye ring (2theta angle between 20.927 and 22.320 associated with the 108 reflection of calcite). At the right bottom, there is a logbook reporting the intensity of peaks found at the Psi scan.

FIGURE 3. Figures $a 1, b 1, c 1$ and $d$ are microphotographic views under crosspolarised light of ostrich, Egyptian vulture, hen and partridge eggshells, respectively. These samples display different microstructural characteristics. Scale bars are equivalent to $100 \mu \mathrm{m}$. The mineral part of the eggshells is constituted by columnar calcite crystal units (palisades) show varying degrees of light extinction due to differences in their orientation. Note that average crystal sizes vary from one eggshell to another. Figures a2, b2, c2 and $d 2$ are the transmission X-ray diffraction patterns of the same eggshells. Figures $a 3, b 3, c 3$ and $d 3$ are the reflection X-ray diffraction patterns of the same eggshells. Ostrich eggshell displays a strong preferential orientation of crystals which is manifested in the reflection spots merging into continuous arcs (indicated by arrows). 
FIGURE 4. Intensity profiles (Psi scans) showing the variation of intensity along a 104 Debye ring in a $2 D$ diffraction pattern in the case of: A) partridge eggshell, B) ostrich eggshell. Hen eggshell is formed by weakly-oriented crystals and its profile consists of isolated peaks. Because of strong orientation of ostrich eggshell a continuous profile is formed instead. C) From the intensity of peaks displayed in A) a calibration curve relating calcite crystal sizes and intensity of reflections such the one depicted here can be determined.

Figure 5. Calculated theta-2theta scans from A) ostrich, B) hen, and C) partridge eggshells from transmission (1) and reflection experiments (2). Note that in the transmission pattern of ostrich eggshell (A1), the strongest calcite 104 reflection is almost absent, while the lower intensity 110 reflection is now the strongest one. Peaks associated to (110) calcite planes are reinforced due to preferential orientation of crystals. In the reflection pattern of highly oriented ostrich egghell (A2), 006 and 104 reflections are strongly reinforced. 


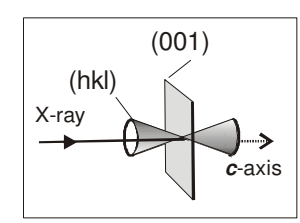

0 1 12 3 4

A

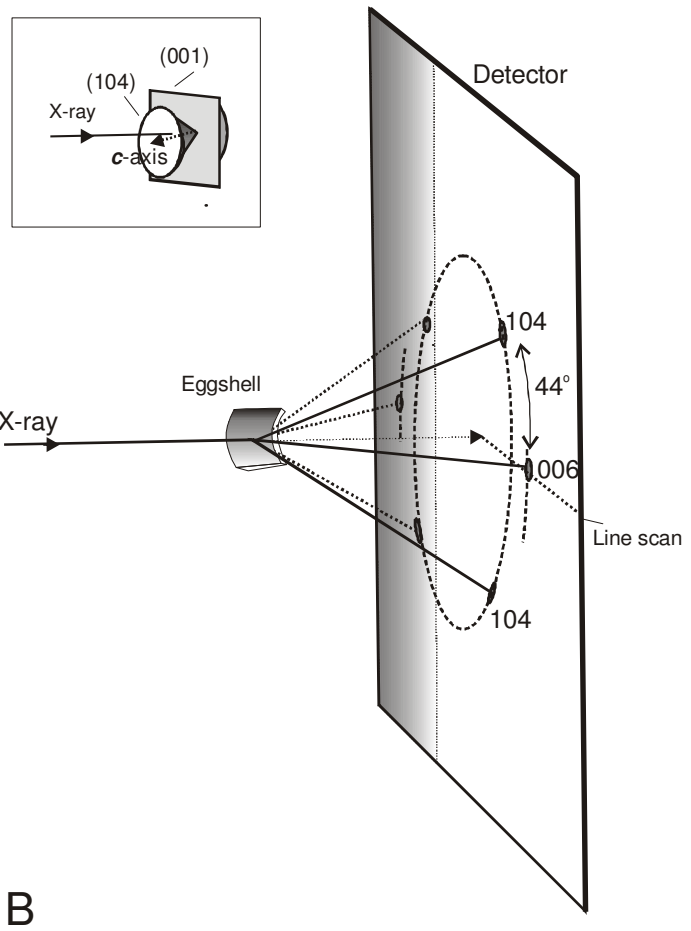

Figure 1 


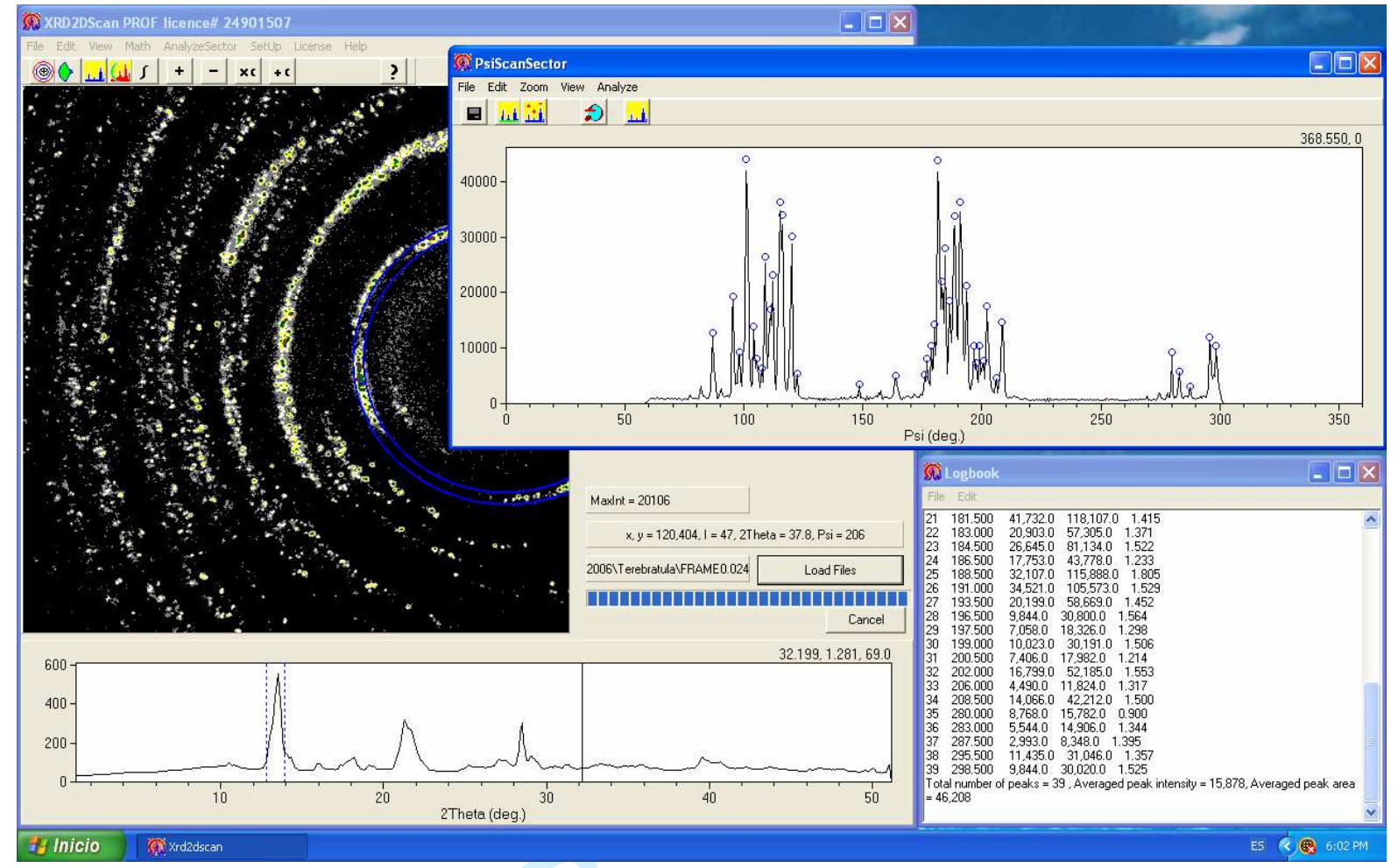

Figure 2 

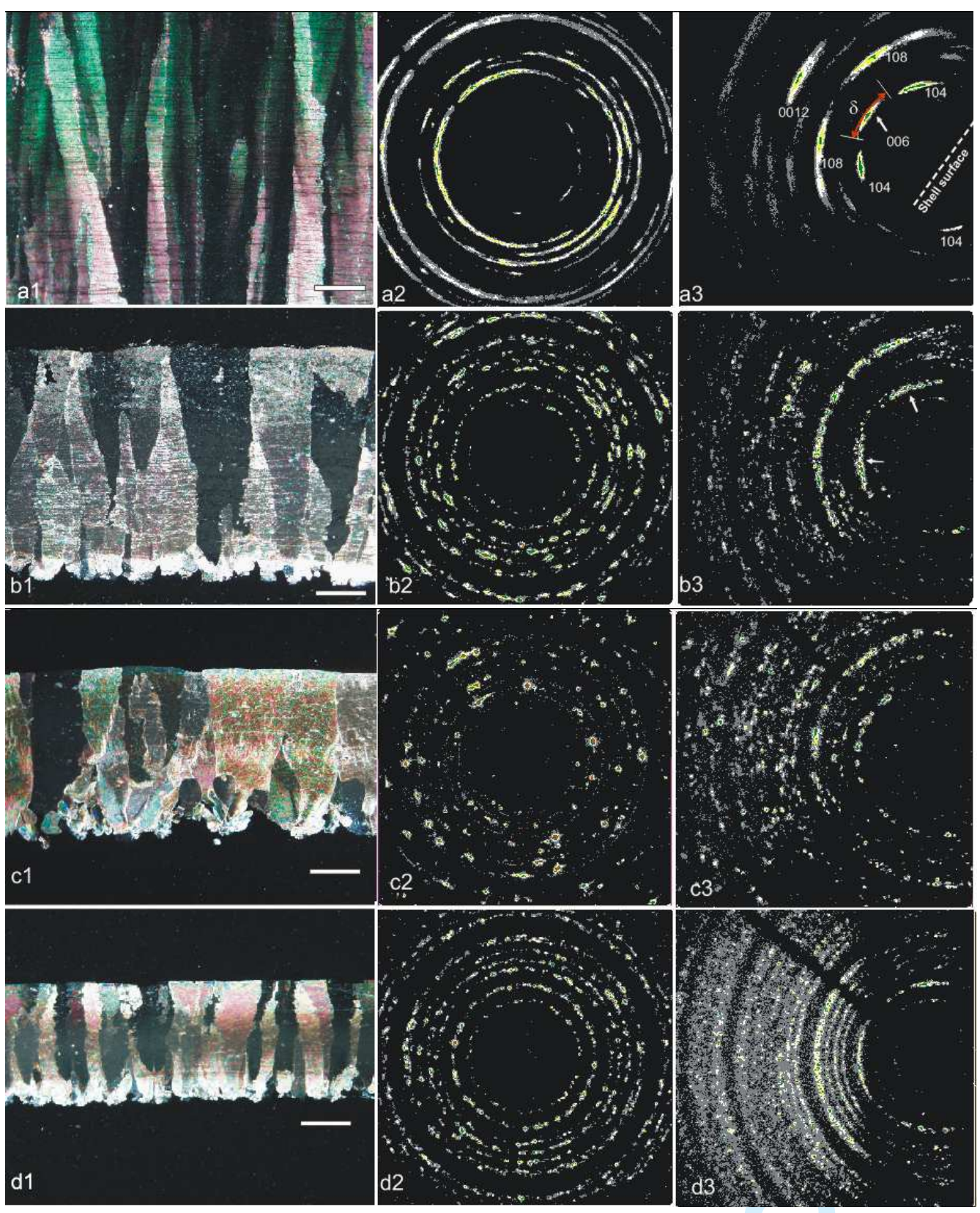

Figure 3 

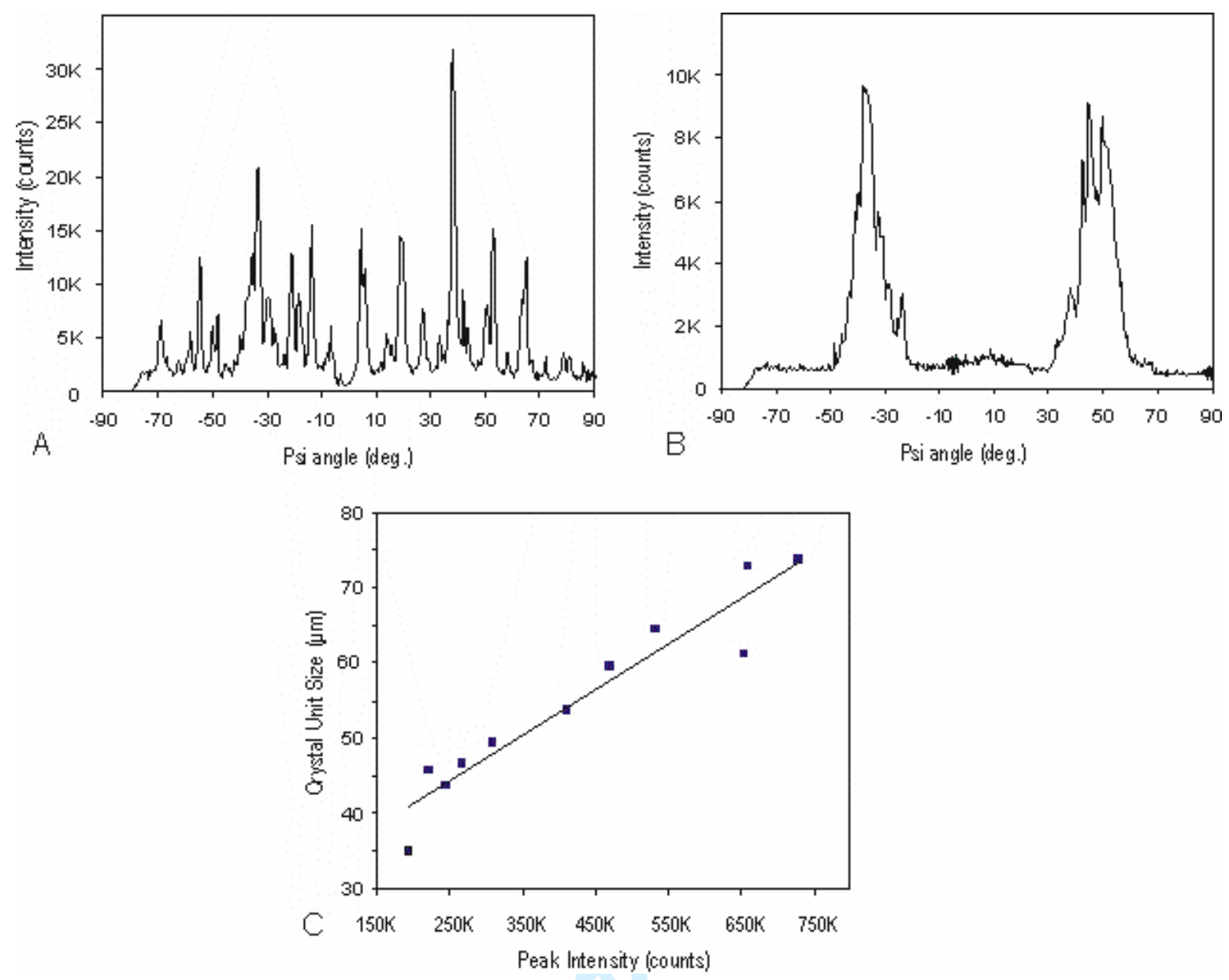

Figure 4 

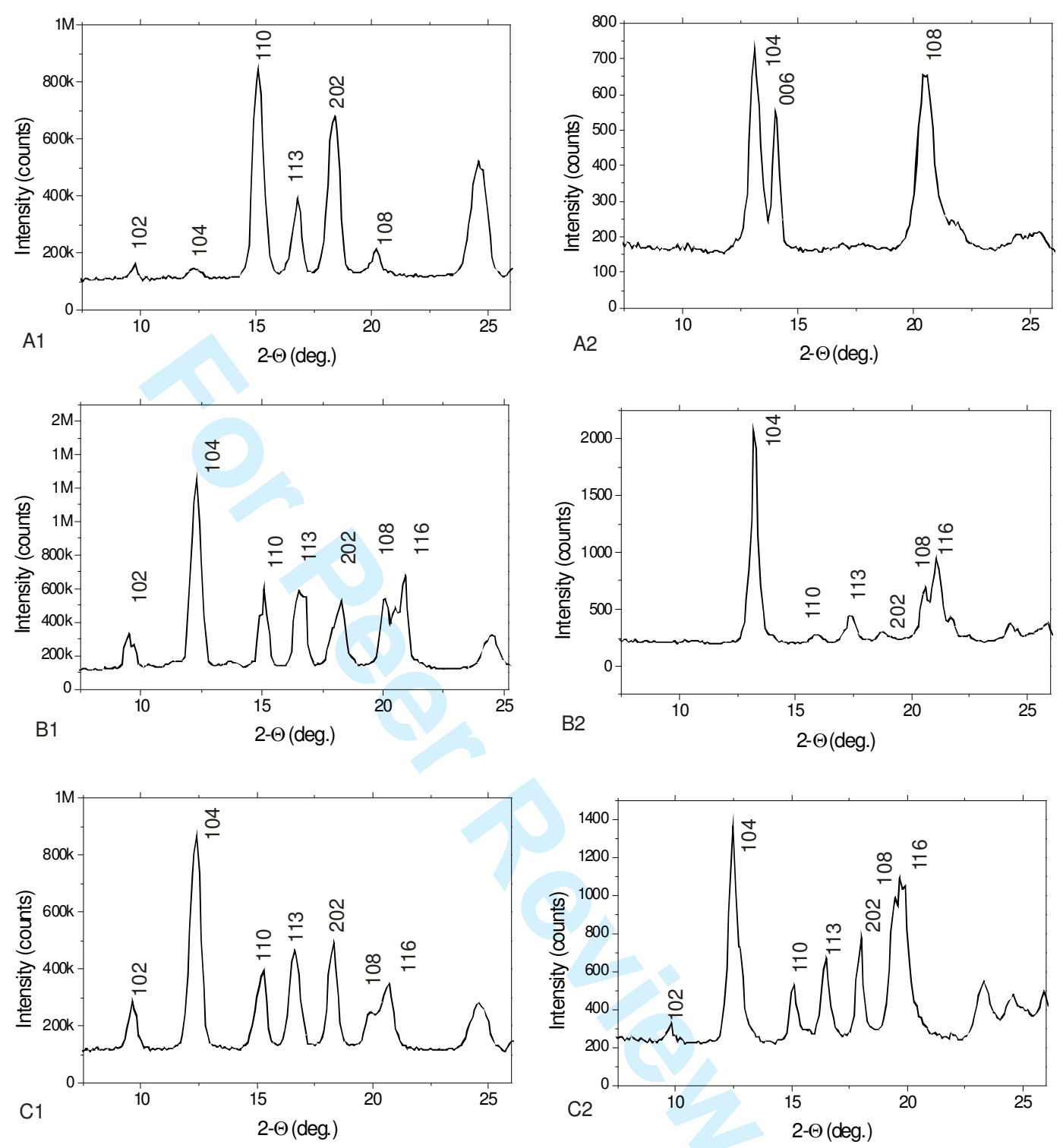

Figure 5 